\title{
ADMINISTRACION DECAPITAL DE TRABAJO UN ENFOQUE IMPRESCINDIBLE PARA LAS EMPRESAS EN EL CONTEXTO ACTUAL
}

\section{Dr. César H. Albornoz - Colaboración: Dr. Miguel Angel Cuello}

\section{RESUMEN:}

El autor analiza aquí "por qué es importante la administración del capital de trabajo para lograr el éxito de la gestión del administrador financiero y por consiguiente, de la empresa". Administrar tal capital se consigue buscando un equilibrio entre la liquidez y la rentabilidad dado que dichas funciones son contrapuestas entre sí. ¿Queda superado el paradigma de que alta liquidez equivale a buena administración financiera? lo ejemplifica mediante cuadros y ejemplos.

\section{ABSTRACT:}

Here the author analyzes "why is it important to manage work capital in order to achieve the success of the financial manager administration and therefore of the company". In order to manage such capital it is necessary to search a balance between liquidity and profitability because those functions are compared among themselves. Is the paradigm of high liquidity equal to good financial management overcome? The author gives charts and examples.

\section{PALABRAS CLAVES:}

Administrar, capital, empresa, equilibrio, liquidez, rentabilidad, trabajo.

\section{KEYWORD:}

Capital, work, company, balance, liquidity, profitability, manage.

\section{DEFINICIONES}

En el presente artículo trataremos de analizar por qué es importante la administración del capital de trabajo para lograr el éxito de la gestión del administrador financiero y por consiguiente de la empresa.

Antes de comenzar con el desarrollo de los temas, precisaremos algunas definiciones para utilizar un lenguaje común.

En un sentido amplio, al capital de trabajo, algunos autores lo denominan también activos de trabajo; involucra la inversión de la empresa en activos corrientes o activos circulantes.

Designaremos capital de trabajo neto a la diferencia entre los activos corrientes y los pasivos corrientes. El término corriente lo entendemos como aquellos bienes o deudas que se establecen dentro del ciclo operativo de la empresa, que normalmente es de un año, y que a su vez coincide con el balance general.

Con estas definiciones, podemos decir que la administración del capital de trabajo se ocupa de la administración de los activos corrientes (caja, bancos, cuentas de cobro, inversiones líquidas, inventarios) y los pasivos corrientes.

Estos activos, que por su naturaleza, cambian muy rápidamente, nos obligan a tomar decisiones y dedicarle un mayor tiempo de análisis, para lograr optimizar 
su uso. El administrador financiero, para cumplir con su objetivo, deberá centrarse en los siguientes puntos:

a) ¿Cuál será el nivel óptimo de la inversión en activos circulantes?

b) ¿Cuál será la mezcla óptima de financiación entre corto y largo plazo?

c) ¿Cuáles serán los medios adecuados

$\mathrm{La}$ administración de capital de trabajo debe permitirnos determinar correctamente los niveles tanto de inversión en activos corrientes como de endeudamiento, que traerán aparejado decisiones de liquidez y de vencimientos del pasivo.

\section{de financiación a corto plazo?}

El capital de trabajo es más divisible que el activo fijo o inmovilizado y por lo tanto, también es mucho más flexible su financiamiento. Las características que distinguen el activo de trabajo del activo fijo son su flexibilidad y la duración de su vida económica.
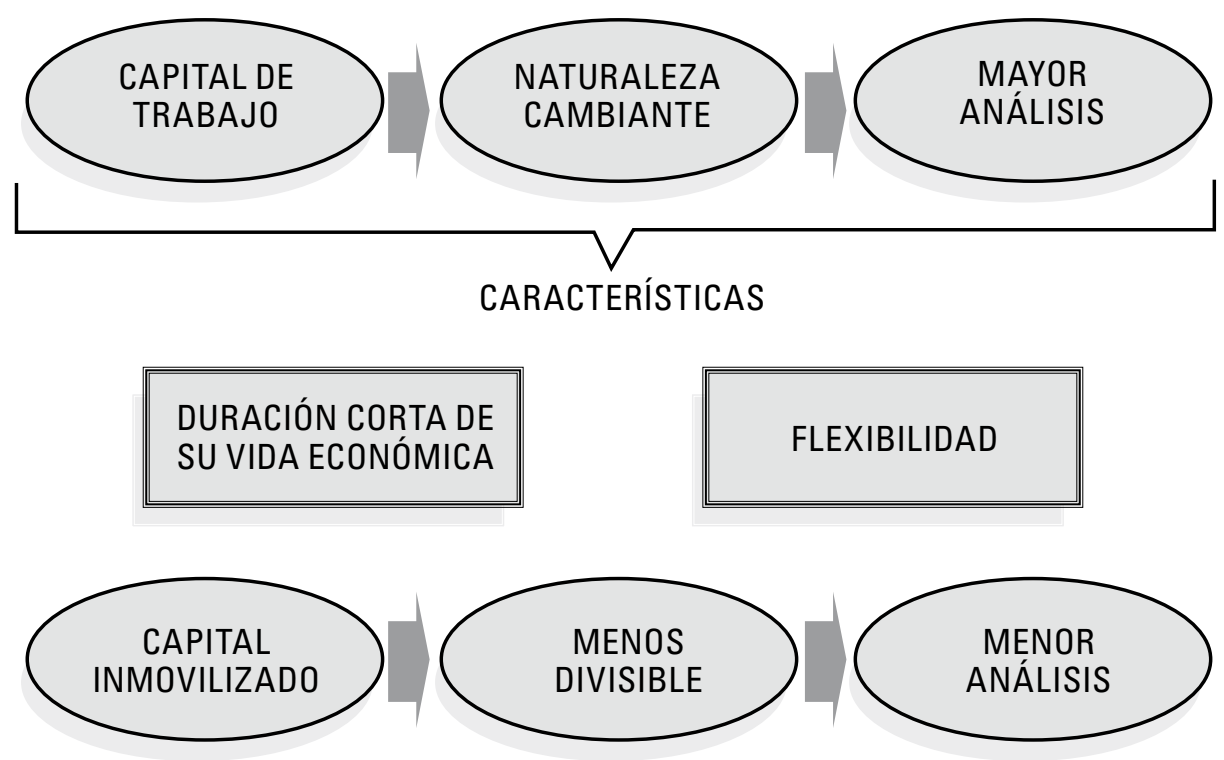

RELACIÓN RIESGO - RENTABILIDAD - LIOUIDEZ EN EL ANÁLISIS DEL CAPITAL DE TRABAJO

Es importante mantener una relación positiva de capital de trabajo. Tal esquema de liquidez nos proporciona un margen de seguridad y reduce la probabilidad de insolvencia y de la eventual quiebra. En oposición a esto, demasiada liquidez trae

\section{RESEÑA DE AUTOR}

César H. Albornoz (cealbornoz@hotmail.com). Docente universitario, Presidente de la Comisión Académica de Estudios sobre Finanzas de Empresas, consultor y autor de Principios de Administración Financiera de empresas. aparejada la subutilización de activos que usamos para generar ventas, y es la causa de la baja rotación de activos y de menor rentabilidad. La administración de capital de trabajo debe permitirnos determinar correctamente los niveles tanto de inversión en activos corrientes como de endeudamiento, que traerán aparejado decisiones de liquidez y de vencimientos del pasivo.

Estas decisiones deben conducirnos a buscar el equilibrio entre el riesgo y el rendimiento de la empresa.

Una política conservadora en la admi- 
nistración de activos de trabajo, traerá aparejada una minimización del riesgo en opoOpuestamente, una política agresiva ponsición a la maximización de la rentabilidad. tos sobre la decisión riesgo-rentabilidad.

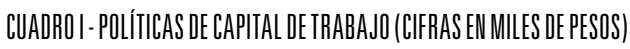

\begin{tabular}{|c|c|c|c|}
\hline RUBROS & Empresa A & Empresa B & Empresa C \\
\hline \multicolumn{4}{|l|}{ ACTIVO } \\
\hline \multicolumn{4}{|l|}{ Activo Corriente } \\
\hline Disponibilidades & 150 & 100 & 50 \\
\hline Créditos por ventas & 450 & 300 & 150 \\
\hline Bienes de cambio & 600 & 400 & 200 \\
\hline Total del activo corriente & 1.200 & 800 & 400 \\
\hline \multicolumn{4}{|l|}{ Activo no corriente } \\
\hline Bienes de uso (neto de amortizaciones) & 800 & 800 & 800 \\
\hline Total del activo no corriente & 800 & 800 & 800 \\
\hline Total del activo & 2.000 & 1.600 & 1.200 \\
\hline \multicolumn{4}{|l|}{ PASIVO } \\
\hline \multicolumn{4}{|l|}{ Pasivo corriente } \\
\hline Deudas comerciales documentadas & 300 & 300 & 300 \\
\hline Deudas sociales y fiscales & 100 & 100 & 100 \\
\hline Total del pasivo corriente & 400 & 400 & 400 \\
\hline \multicolumn{4}{|l|}{ Pasivo no corriente } \\
\hline Deudas comerciales & 200 & 150 & 100 \\
\hline Deudas bancarias & 400 & 250 & 100 \\
\hline Total del pasivo no corriente & 600 & 400 & 200 \\
\hline Total del pasivo & 1.000 & 800 & 600 \\
\hline \multicolumn{4}{|l|}{ PATRIMONIO NETO } \\
\hline Capital suscripto & 700 & 500 & 300 \\
\hline Resultados acumulados & 300 & 300 & 300 \\
\hline Total del patrimonio neto & 1.000 & 800 & 600 \\
\hline Total del pasivo más patrimonio neto & 2.000 & 1.600 & 1.200 \\
\hline
\end{tabular}




\begin{tabular}{|c|c|c|c|}
\hline \multicolumn{4}{|l|}{ CUADRO DE RESULTADOS } \\
\hline Ventas netas & 2.000 & 2.000 & 2.000 \\
\hline Costo de mercaderías vendidas & $(1.000)$ & $(1.000)$ & (1.000) \\
\hline Margen bruto & 1.000 & 1.000 & 1.000 \\
\hline Gastos de administración & $(110)$ & $(110)$ & $(110)$ \\
\hline Gastos de fabricación & $(250)$ & (250) & (250) \\
\hline Gastos de comercialización & $(280)$ & (280) & (280) \\
\hline Utilidad antes de intereses e impuestos & 360 & 360 & 360 \\
\hline Gastos financieros & (60) & (40) & (20) \\
\hline Utilidad antes de impuestos & 300 & 320 & 340 \\
\hline Impuesto a las gananci as (50\%) & $(150)$ & (160) & (170) \\
\hline Utilidad Neta & 150 & 160 & 170 \\
\hline
\end{tabular}

En el ejemplo del cuadro I las tres compañías operan en el mismo sector industrial, pero se diferencian en sus políticas de administración de capital de trabajo.

La empresa A es la que emplea una política muy conservadora, la empresa $\mathbf{B}$ sigue una política promedio para la industria y la empresa $\mathbf{C}$ plantea una política de capital de trabajo muy agresiva.
Como podemos apreciar en el cuadro I, las políticas sobre administración de capital de trabajo tienen diferencias sustanciales entre las tres empresas.

Para visualizar el manejo del capital de trabajo de cada una, se analizaron los siguientes ratios:

\begin{tabular}{|l|c|c|c|}
\hline \multicolumn{1}{|c|}{ RUBROS } & Empresa A & Empresa B & Empresa C \\
\hline Indice de liquidez & 3,0 & 2,0 & 1,0 \\
\hline Rotación del activo corriente & 1,67 & 2,5 & 5,0 \\
\hline $\begin{array}{l}\text { Relación pasivo corriente a pasivo no } \\
\text { corriente }\end{array}$ & 0,67 & 1,0 & 2,0 \\
\hline Rendimiento sobre el capital (ROE) & $15 \%$ & $20 \%$ & $28 \%$ \\
\hline
\end{tabular}

ESTRATEGIA CONSERVADORA

La empresa A presenta una la política onerosa si la analizamos desde el punto de vista de rendimiento del capital, al mantener una elevada proporción de activos líquidos. Además como se puede apreciar la rotación de los activos totales $(2000 / 2000=1)$ es lenta en función de la alta 
inversión en activos corrientes usados para obtener ventas. Si esta empresa hubiera realizado otras combinaciones más agresivas en las relaciones entre activos corrientes y pasivos corrientes, podría en primer lugar, haber mejorado el margen de utilidad neta y en segundo lugar hubiera rotado más rápidamente el activo corriente.

\section{ESTRATEGIA AGRESIVA}

En cambio, la estrategia de la empresa $\mathrm{C}$, netamente agresiva, produjo una rotación de activos más elevada y a su vez, un rendimiento sobre el capital mayor. La tasa de rentabilidad sobre el capital fue mayor a costa de un mayor riesgo y una menor posición de liquidez.

Como podemos ver estas decisiones están influidas por la compensación entre el riesgo y la rentabilidad. Si lo vemos desde el punto de vista de los activos corrientes, cuanto menor sea la proporción de éstos, mayor será la rentabilidad sobre el total de las inversiones de la empresa. en los casos de la empresa $\mathbf{C}$ sobre la empresa $\mathbf{A}$. Si lo analizamos desde el aspecto de los pasivos, la rentabilidad se mide por el costo de las distintas fuentes de financiamiento y al uso durante los períodos en que no se los utiliza. Normalmente los costos explícitos de las deudas a corto plazo son menores que los de las de largo plazo, o sea, que cuanto mayor sea la proporción del uso de las deudas de corto plazo, mayor será la rentabilidad de la empresa. Además la utilización de deudas de corto plazo, permite disminuirlas en aquellos momentos en que por estacionalidad o por incrementos en los ingresos no las necesitamos, esto también hará obtener una mayor rentabilidad.
En función de lo expresado, será conveniente tener una baja proporción de activos corrientes o circulantes sobre los activos totales y una alta proporción de pasivos corrientes sobre el endeudamiento total. Esto producirá obviamente un bajo nivel de capital de trabajo.

El análisis de las políticas de capital de trabajo nos pone frente a ciertos interrogantes, que trataremos a lo largo de este trabajo poder despejar. Por ejemplo:

(a) La razón de capital de trabajo, ¿es la adecuada para asegurar el máximo de rentabilidad sin poner en peligro la liquidez?

(b) Suponiendo que esta es la proporción adecuada, ¿la mezcla de los activos corrientes es la más apropiada para garantizar la máxima rentabilidad dados ciertos requerimientos de liquidez?

(c) El capital de trabajo neto, ¿es el que nos asegura el mayor volumen de beneficios?

(d) Por último, ¿en qué momento conviene invertir o desinvertir en activos corrientes, cambiar la mezcla de los mismos o cambiar las fuentes de financiamiento?

\section{CICLO OPERATIVO: DINERO - MERCADERÍA - DINERO}

Como expresamos al comienzo, el capital de trabajo le permite a la empresa cumplir con su ciclo operativo: esto implica que transforma el dinero en mercadería para venderla y que nuevamente la convierta en dinero. Este ciclo es aquel que demora el dinero en transformarse nuevamente en dinero.

\section{Será}

conveniente tener una baja proporción de activos corrienteso circulantes sobre los activos totales y una alta proporción depasivos corrientes sobre el endeudamiento total. 
Si lo expresamos gráficamente sería:

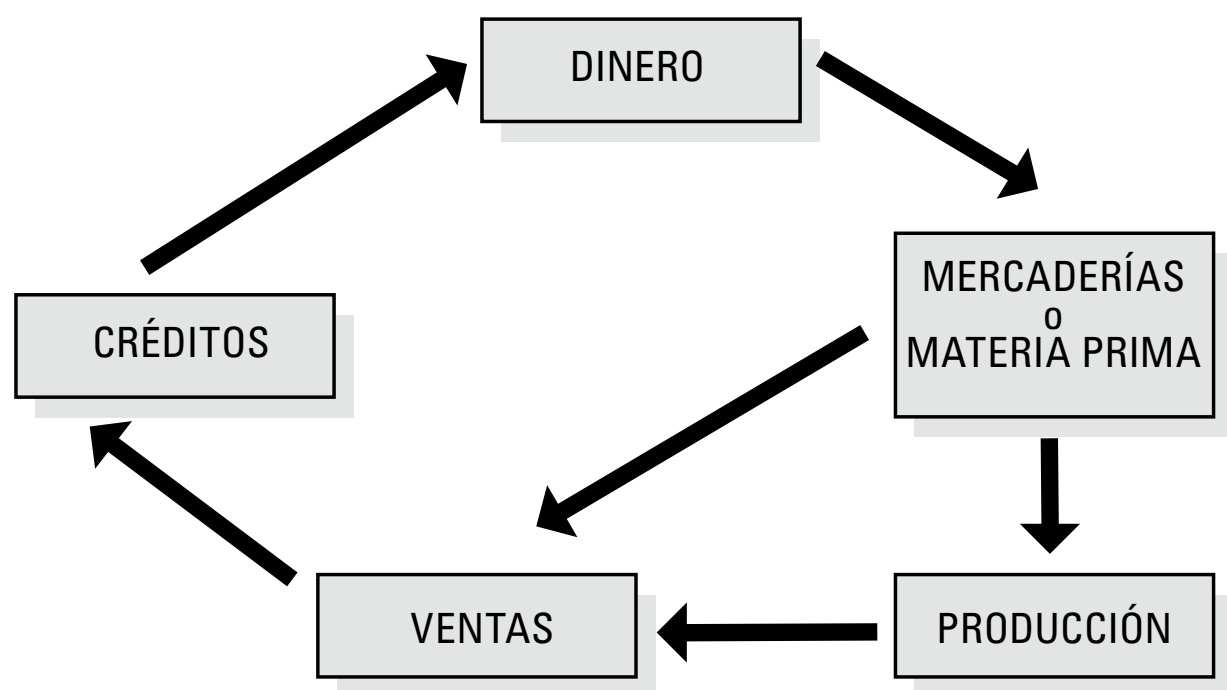

Estos ciclos no necesariamente serán iguales para todas las empresas, tanto en su constitución como en su duración, dado que estarán en función de la cantidad de etapas que deberán cumplir para cerrar el círculo.

Por ejemplo, para una empresa que se dedica a la reventa de productos, el ciclo sería:

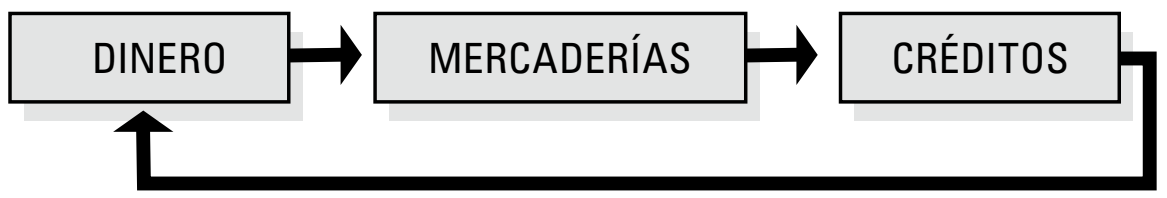

En cambio en una empresa industrial, el ciclo sería:

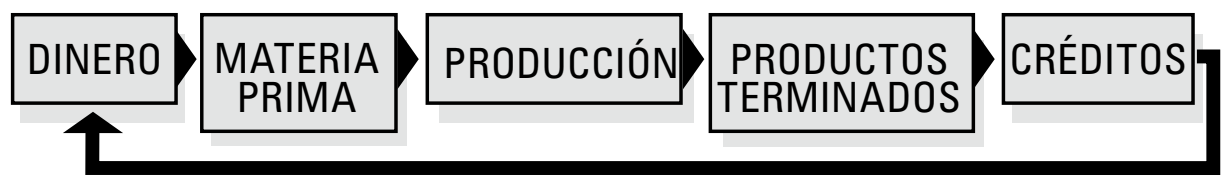

La distinta duración de los ciclos de estas empresas, no implica necesariamente que la empresa industrial requiera una mayor inversión en capital de trabajo, sino que la magnitud de este va a depender de otras variables, como por ejemplo, la duración de cada ciclo y la cantidad de superposiciones o solapamientos que estos tengan, el nivel de actividad con que operará la empresa y el costo del producto, entre otras. 
Este ciclo en la empresa es de vital importancia para la formulación de políticas y estrategias de la administración del capital de trabajo.

De lo expresado anteriormente, podemos inferir que tanto el ciclo operativo como la rotación del capital de trabajo son términos equivalentes, dado que cuanto menor sea el ciclo dinero-mercaderíadinero, mayor será la rotación del capital de trabajo, o sea menor la inversión en capital de trabajo.

\section{CARACTERISTICAS PARtiCULARES DE LOS CICLOS}

Si analizáramos los componentes que intervinieron en cada ciclo, veríamos que hay dos de ellos que constituyen excedentes financieros a corto plazo, tal cual son las amortizaciones o costos fijos no erogables y las utilidades.

Decimos esto, puesto que las amortizaciones, si bien por lo general proporciona una fuente de fondos, no son una partida de caja, por lo que no se refleja como una fuente de efectivo. Sin embargo, ésta con frecuencia sirve para disminuir la salida de fondos, en la forma de pagos del impuesto a las ganancias.

Las empresas normalmente aplican fondos para activos fijos, que con el tiempo esperan recuperar mediante la venta de sus productos. Cuando se fija el precio de venta, la empresa busca recuperar todos los costos inmediatos (materias primas, mano de obra, energía, etcétera) y aquellos que fueron desembolsados bastante tiempo atrás (amortizaciones). Cuando afirmamos que la amortización es una fuente de fondos, estamos diciendo que los fondos son generados en la medida en que los ingresos por ventas excedan a los gastos que utilizan esos fondos.
Una retracción de las ventas disminuirá la cantidad de ciclos que se realizan y por lo tanto aumentará la necesidad de inversiones adicionales en capital de trabajo.

\section{DURACIÓN DE LOS CICLOS}

La duración del ciclo dinero-mercadería-dinero y la cantidad de solapamientos que se producen durante su prolongación en el tiempo, condicionan la magnitud requerida de capital de trabajo; estas dos variables además, se encuentran íntimamente relacionadas con el sector de la economía en que opera la empresa, la tecnología con que se dispone y las decisiones políticas que toma la empresa para lograr el desarrollo de su actividad.

Sin lugar a dudas el ciclo operativo de un astillero es diferente al de un fabricante de partes para automóviles, como así también es diferente el grado de tecnología que se emplea en la industria electrónica con respecto a un fabricante artesanal de zapatos.

Como puede observarse la duración del ciclo está relacionada con el sector de la economía y con el nivel tecnológico; si tenemos dos empresas que desarrollan actividades en el mismo sector económico, es muy probable que las diferencias en cuanto a la duración del ciclo operativo de una empresa con respecto a la otra, estén dadas por el nivel tecnológico de cada una de ellas, como por las decisiones de política interna que se tomaron para definir el desarrollo de su operatoria.

Es conveniente analizar primeramente el caso más simple, que es el de una empresa que inicia su ciclo operativo sólo cuando haya terminado con el ciclo inmediato anterior, es decir que no produce superposiciones de ciclos. En este caso el requerimiento del 
capital de trabajo queda supeditado a la cantidad de ciclos que se efectúen en la unidad de tiempo que se analice (año, semestre, trimestre, etcétera). La empresa realizará una mayor o menor cantidad de ciclos operativos, en un lapso de tiempo determinado, dependiendo de la duración de cada uno de ellos, cuanto más largo sea el ciclo en su duración, menor será la cantidad de ellos en el período que se analice y viceversa.

Una empresa cuyo ciclo operativo dura 2 meses, producirá 6 ciclos en un año, en tanto que si el ciclo se alarga a 3 meses, realizará sólo 4 ciclos en el año.

Al no existir superposiciones de ciclos operativos el monto del capital de trabajo está determinado por la cantidad de fondos que se necesitan durante el ciclo hasta su conclusión, independientemente de la prolongación de éste.

Para el supuesto anterior, de 6 ciclos en el año, si el capital requerido por cada ciclo es de $\$ 70$, se requerirán $\$ 420$ en el año, pero al no producirse superposiciones, se necesita sólo $\$ 70$ por cada ciclo operativo. Para el caso de alargarse el ciclo a 3 meses, se reducirán la cantidad de ciclos en el año, ya que pasarán a ser 4 ciclos, pero al no modificarse la necesidad de capital se requerirán \$280 para el año, mientras que permanece necesitando sólo $\$ 70$ por cada ciclo operativo igual que en el caso anterior.

El análisis se complica cuando se producen superposiciones o solapamientos de ciclos, es decir que la empresa empieza un ciclo o más, sin haber finalizado el inmediato anterior. Es importante aclarar que cada ciclo que se comienza implica una nueva inversión.

Ante esta situación, cabe destacar que se producen dos aspectos fundamentales para la determinación del capital de trabajo; uno es la duración del ciclo operativo de la empresa (dinero-mercadería-dinero), y el otro es el período de tiempo que existe entre el inicio de cada uno de los ciclos.

Supongamos una situación en la cual el capital de trabajo necesario para realizar un ciclo es de $\$ 25$, la duración del mismo es de 18 días y se produce el inicio de un nuevo ciclo cada 3 días:

- Cantidad de solapamientos: 5

- Capital necesario por ciclo: \$ 25

- Capital de trabajo requerido por los solapamientos: \$ 125

- Capital de trabajo total (incluido ciclo inicial + solapamientos): $\$ 150$

Otra situación que puede ocurrir es que los ciclos comiencen cada 3 días, pero que la duración del ciclo se prolongue a 30 días, con lo cual se produce un cambio sustancial, quedando:

- Cantidad de solapamientos: 9

- Capital necesario por ciclo: \$ 25

- Capital de trabajo requerido por los solapamientos: \$ 225

- Capital de trabajo total (incluido ciclo inicial + solapamientos): $\$ 250$

Como puede observarse en estas dos situaciones planteadas precedentemente, el monto total de capital de trabajo requerido se obtiene de la multiplicación de la cantidad de solapamientos o superposiciones por el capital de trabajo necesario para cada ciclo.

En resumen podemos decir que cuanto mayor sea la duración del ciclo operativo y mayor la cantidad de solapamientos que se produzcan, se obtendrá como resultado un requerimiento de capital de trabajo mayor. 
POLÍTICAS EN LA ADMIIIISTRACIÓN DECAPITAL DE TRABAJO

Como habíamos expresado al comienzo de este trabajo, las políticas inherentes al capital de trabajo involucran la administración de activos corrientes o circulantes de la empresa, también la obtención de los medios más adecuados para financiar estos activos.

La habilidad de la empresa para poder administrar de una forma apropiada los activos corrientes y la estructura de vencimientos del pasivo determinará qué tanto pueda sobrevivir en el corto plazo. Siempre y cuando una parte importante de los activos circulantes tenga el carácter de permanente, las necesidades de financiamiento deberán ser consideradas por períodos más prolongados.

En este punto nos ocuparemos de las políticas referidas a la administración de activos corrientes, o sea, disponibilidades, inversiones transitorias, créditos y administración de inventarios, tratando en cada uno de ellos de visualizar los elementos y las restricciones, y así hacer un uso óptimo de ellos.

\section{a) Política de disponibilidades $\mathrm{e}$ inversiones}

En la administración del efectivo y las inversiones transitorias, lo más importante es lograr un volumen tal que nos permita tener un determinado grado de liquidez, sin que ésta afecte la rentabilidad, o sea, la maximización de utilidades de la firma.

Una de los primeros objetivos del administrador financiero en cuanto al manejo del efectivo, será la sincronización entre los flujos de entrada y salida, dado que esto permitirá a la empresa cumplir satisfactoriamente con el ciclo operativo.

Como mencionan Weston y Copeland en su libro titulado Finanzas en Administración de Mcgraw-hill 1995, uno de los aspectos importantes en la administración de la posición de liquidez de la empresa, consiste en desarrollar sistemas eficientes para la administración de los flujos de entrada y salida de efectivo.

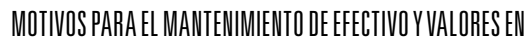 CARTERA}

Los anteriores autores se preguntan ${ }_{i}$ por qué se debe mantener efectivo y valores negociables (o inversiones transitorias)? y dan cuatro motivos principales.

El primero de ellos es el transaccional, que consiste en permitirle a la empresa poder realizar sus operaciones habituales (comprar, vender).

El segundo motivo es el preventivo, que tiene que ver con la seguridad y se relaciona con la estimación que realizó la empresa sobre los flujos de ingresos y egresos.

El otro motivo tiene que ver con la satisfacción de necesidades futuras, esto generalmente ocurre en forma temporal cuando por razones de estacionalidad de ventas o de compras nos vemos en la necesidad de acumular efectivo o inversiones transitorias para hacer frente a esos requerimientos.

Por último, el requerimiento de saldo compensadores, esto tiene relación con los saldos bancarios inmovilizados a consecuencia de acuerdos por créditos preexistentes, y que generalmente son saldos mínimos que la empresa se compromete a mantener en su cuenta bancaria. El mantenimiento de estos saldos es lo que denominamos activos corrientes de tipo permanente, lo mismo que los efectivos mantenidos en forma de cajas chicas o fondos fijos con asignación específica.
En este punto nos ocuparemos de las políticas referidas a la administración de activos corrientes, o sea, disponibilidades, inversiones transitorias, créditos $y$ administración de inventarios, tratando en cada uno de ellos de visualizar los elementos y las restricciones, y asíhacer un uso óptimo de ellos. 
RAZONES PARA EL MANTENIMIENTO DE ACTIVOS LíquIOOS

Normalmente una empresa mantiene dinero en efectivo y en inversiones transitorias para cubrir las transacciones que realiza. El mantenimiento de fondos excedentes para fines preventivos, en realidad representa un inventario de seguridad, que depende de su volumen y tienen asociado un costo de inmovilización que repercute en la rentabilidad de la empresa e inversamente acotan el riesgo de insolvencia.

Por el contrario, si el monto de los fondos de efectivo fuera insuficiente o con una distribución errática en el tiempo, nos encontraríamos que las operaciones normales de la empresa podrían verse interrumpidas, con los consiguientes costos asociados.

Como podemos observar, el análisis de las decisiones de administración de efectivo $\mathrm{y}$ de inversiones transitorias requiere de un minucioso análisis y del uso de herramientas que permitan optimizar el cálculo de dichos saldos.

\section{aSpeCtos para tener en CUENTA}

Con el fin descrito anteriormente, el administrador financiero deberá desarrollar un sistema de proyección diaria de orígenes y necesidades de fondos con el fin de tener un adecuado sistema de cobertura. Como complemento a estas proyecciones, deberá llevar un sistema de control de caja diaria para comparar la realidad con las estimaciones y efectuar las correcciones necesarias.

También deberá complementar estas proyecciones con un adecuado seguimiento de las conciliaciones bancarias, con el fin de detectar tanto aquellos movimientos registrados por el banco y que no lo tuviéramos registrados en nuestros libros (por ejemplo débitos por gastos e intereses, depósitos, etc.), como los cheques emitidos y no cobrados y los depósitos no acreditados, y todas aquellas partidas que todavía se encuentran pendientes de ser acreditadas o debitadas. Con respecto a las que están pendientes de ser acreditadas, es importante previamente efectuar un análisis de los plazos y los costos de los valores al cobro en los distintos bancos donde trabaja la empresa, con el objetivo de acortar los plazos de disponibilidad de los mismos y los costos asociados; asimismo una vez depositados debemos realizar un seguimiento muy estricto de las fechas de acreditación.

Otro elemento a tener en cuenta con respecto a los cheques emitidos por la empresa, son aquellos que todavía se mantienen en cartera sin retiro por los beneficiarios, dado que los mismos ya han sido descontados de los saldos bancarios de libros que lleva la empresa, y que si esta tiene una adecuada programación de las fechas de pago, se puede optimizar la utilización de los mismos.

Con respecto a las inversiones transitorias, las empresas las utilizan en lugar del dinero en efectivo para realizar determinadas colocaciones de tipo temporal.

Como mencionamos anteriormente, muchas veces los bancos requieren determinada inmovilización de fondos en cuenta corriente para garantizar las cuotas de un préstamo entre otros conceptos; por lo tanto, es mucho más conveniente colocar esos fondos en inversiones transitorias que generan un interés compensatorio en parte del costo de la inmovilización.

En otras ocasiones, las empresas necesitan acumular fondos líquidos; por ejemplo, para pagar una cuota de un préstamo o 
de una obligación negociable. Esos fondos conviene colocarlos en inversiones transitorias de rápida disponibilidad que nos permita, al vencimiento, utilizarlos.

Como vimos, las políticas sobre la liquidez de la empresa varían según las circunstancias y las necesidades de cada momento, y a su vez, están íntimamente ligadas con el ramo de cada sector.

En resumen, la administración de disponibilidades e inversiones conduce a considerar el equilibrio entre el riesgo de la liquidez y el rendimiento que enfrenta cualquier administrador financiero. Asimismo, una administración eficiente comienza entendiendo perfectamente el ciclo de operaciones de corto plazo de la empresa, unido a los presupuestos de caja; ayuda a mantener un equilibrio óptimo en los niveles de liquidez, la consecuente disminución del riesgo de insolvencia y el incremento de la rentabilidad.

\section{b) Políticas de créditos y cobranzas}

Las políticas de administración de créditos implican un equilibrio entre el riesgo y la rentabilidad. O sea, la extensión de condiciones de crédito a clientes marginales compromete fondos y pone en peligro la propia capacidad de pagar cuentas, en tanto facilita la venta de los productos y mejora los resultados.

El papel que juega el administrador financiero en la administración de las políticas de créditos y cobranzas es dinámico, ya que trae aparejado ajustes frecuentes a los estándares de crédito, a los términos de crédito y a las politicas de cobranzas.

Normalmente la mayor proporción de la inversión en activos corrientes lo constituyen las cuentas de cobro derivadas de los incrementos de ventas y el alargamiento de los plazos de cobranza. Esto último se produce generalmente en los períodos de recesión económica, cuando los pequeños clientes descansan en el financiamiento de los proveedores, contrariamente a lo que se produce cuando hay crecimiento económico cuando hay una expansión de las ventas y los plazos de cobranzas se vuelven más estables.

De la misma manera que cualquier activo corriente, las cuentas de cobro también deben ser consideradas una inversión. En este punto debemos tener en cuenta si el rendimiento que podemos obtener de este activo supera la ganancia de otras inversiones y cuál es el riesgo y la liquidez asociados con este rendimiento.

Al analizar el tema de las cuentas de cobro, consideremos el otorgamiento de créditos como uno de los puntos claves dentro de la administración de capital de trabajo.

Recordemos las siguientes variables con respecto a las políticas de otorgamiento de créditos en relación con el objetivo de maximización de utilidades.

1. Estándares de crédito

2. Términos de crédito

3. Políticas de cobranzas

\section{Estándares de crédito}

La empresa debe definir la naturaleza del riesgo de crédito que está dispuesta a asumir, que se basa en el historial del cliente, de su situación financiera y de las referencias que pueda recabar.

Para ello, efectuará el análisis del crédito, que implica las cinco condiciones que debe reunir un crédito. El análisis comienza con el carácter, que es una evaluación de la dis- 
posición para pagar las cuentas de cobro. La capacidad representa la habilidad de la empresa para cumplir con sus obligaciones mediante el flujo proveniente de sus operaciones. En este punto el administrador financiero estará interesado en las relaciones de liquidez del crédito y en los flujos de efectivo del cliente. Es importante, también evaluar el capital del cliente en el caso de que éste tenga dificultad para cubrir sus obligaciones con las operaciones normales. La relación de deuda a activos y otros indicadores que marcan la posición del capital del cliente deben analizarse en la evaluación del mencionado capital. También las condiciones que existen y que se esperan en términos de actividad económica pueden ser de significativa importancia al analizar a los solicitantes de créditos. Otro aspecto importante que constituye el análisis del crédito es la garantía que eventualmente podría ofrecer el cliente.

El administrador financiero deberá estar dispuesto a ajustar los estándares de crédito a los cambios en el ciclo comercial, si encuentra que tales políticas de crédito son para mejorar el comportamiento de la empresa.

\section{Términos comerciales}

Los términos comerciales tienen un fuerte impacto sobre el volumen del saldo de créditos por ventas. Si por ejemplo, una empresa realiza ventas a crédito diarias del orden de los $\$ 15.000$ y el término de cobranzas es de 30 días, el promedio del saldo de los créditos por ventas sería de \$ 450.000. Esta situación se mantendría en promedio de esta forma si todos los clientes abonaran en el plazo estipulado, pero si los créditos por ventas se cobraran a los 60 días en lugar de los 30 que estipula la condición de venta, el saldo promedio de los créditos por ventas sería de $\$ 900.000$, y por lo tanto, la empresa requeriría un financiamiento mayor para cubrir el adicional de inversión en créditos por ventas.

También el administrador financiero deberá establecer términos comerciales con relación a la extensión del período de crédito y al grado hasta el cual se ofrezcan descuentos por pago de contado a los clientes que operan a crédito, considerando cómo afecta esta decisión en la rentabilidad de la empresa y analizando las restricciones de liquidez en función del ciclo operativo de la empresa.

Debemos tener en cuenta que las políticas de términos comerciales no son independientes de las políticas de estándares de crédito.

\section{Política de cobranza}

La función de cobranza constituye un área muy importante dentro de la política de créditos de la empresa. Diversas herramientas deberán utilizar el sector de cobranzas con el fin de efectuar un seguimiento de las políticas de crédito fijadas. Entre ellas podemos mencionar:

- Indice de incobrables sobre las ventas a crédito: un incremento de este indicador, podría ser una señal de que se esté realizando una política agresiva de ventas en el mercado o que se estén otorgando créditos a clientes poco solventes.

- Período promedio de cobranza:

$$
\begin{gathered}
\text { Créditos por ventas x } 365 \\
\text { Total ventas a crédito }
\end{gathered}
$$


Un aumento de este índice no indicaría: a) una mala gestión de cobranza o administración del crédito o b) que la empresa decidió ampliar los términos de sus créditos.

- Análisis de antigüedad de saldos: Con este análisis podremos observar la demora en ser cobradas las cuentas de cobro y poder tomar medidas correctivas para agilizarlas.

La implementación de políticas de cobranzas también tiene su impacto en la rentabilidad y el rendimiento de la empresa para sus accionistas. Si la compañía no es estricta en hacer cumplir sus condiciones de crédito, entonces el período real de cobranza será significativamente mayor al establecido y por supuesto, más oneroso. Por otra parte, una política de cobranza rígidamente administrada puede dar como resultado una pérdida de ventas y una mala predisposición de los clientes. Las condiciones económicas del mercado afectarán las políticas de cobranzas.

\section{c) Política de administración y control de inventario}

Este tema merece una particular atención en tres aspectos. Primero, el inventario comprende generalmente un importante segmento de los activos totales de la empresa. Segundo, siendo el inventario el menos líquido de los activos corrientes, los errores en su administración no se pueden solucionar con rapidez. Tercero, los cambios en los niveles de inventario tienen importantes efectos económicos.

El objetivo del administrador financiero es minimizar los costos asociados con la administración de los inventarios. Por un lado, un nivel de inventarios bajo reduce los costos de financiamiento y de manteni- miento de inventario y, por otra parte, un inventario exiguo puede dar como resultado costo de escasez (pérdida de ventas) y costos elevados de ordenamiento del mismo.

Generalmente, dependiendo de la clase de empresas, tendremos diferentes tipos de inventarios: de materias primas, de producción en proceso y de productos terminados.

El nivel de inventario de materias primas se ve influido por la estacionalidad, por las fuentes de aprovisionamiento y por la programación de las compras. El de producción en proceso estará afectado por la duración del proceso de producción. El inventario de productos terminados, por la coordinación entre la producción y las ventas.

El administrador financiero, tendrá en cuenta el nivel de actividad económica, dado que el periodo de conversión del inventario en ventas se alarga en épocas de recesión. Por lo tanto, es muy importante mantener y controlar estrictamente el inventario anticipándose a estos procesos y por el tiempo que dure.

El administrador financiero, debe esforzarse por mantener los tiempos de conversión del inventario y equilibrar los costos del mismo cuando decline la actividad económica, así como estar listos para satisfacer la demanda de las ventas cuando se inicie la recuperación.

Gran cantidad de análisis se ha realizado respecto de determinar el nivel óptimo de inventarios, lotes óptimos de compra o producción, niveles de consumo y otras consideraciones similares.

Cuando evaluamos un modelo de inventario, debemos analizar los dos costos básicos asociados con el inventario: los costos para mantener las existencias en inventario y 
Los costos para mantener inventarios incluyen los intereses sobre los fondos invertidos en el inventario, el costo del espacio para almacenamiento, las primas de seguro y los gastos respecto al manejo de materiales. los costos concernientes a pedidos. Mediante un análisis cuidadoso de estas variables, podremos determinar el tamaño óptimo de pedido, de manera que se minimicen los costos.

Los costos para mantener inventarios incluyen los intereses sobre los fondos invertidos en el inventario, el costo del espacio para almacenamiento, las primas de seguro y los gastos respecto al manejo de materiales. Existe también un costo implícito asociado a los riegos de obsolescencia y cambios inesperados en los precios. Cuanto mayor sea el tamaño de la orden, mayor será el promedio de inventario que tendremos en existencia y mayores serán los costos por mantenimiento. Otro elemento que debemos considerar es el costo de ordenar y procesar el inventario. Si mantenemos un nivel promedio bajo en inventario, nos veremos obligados a ordenar muy seguido $y$, por lo tanto, nuestros costos de ordenar serán muy elevados.

Por lo tanto, para poder definir una política adecuada de inventarios, debemos conocer los costos asociados con el mantenimiento de inventarios.

En el siguiente esquema una enumeración de los principales costos:

\begin{tabular}{|l|l|l|}
\hline Costos de mantenimiento & Costos de ordenamiento & \multicolumn{1}{|c|}{ Costos de seguridad } \\
\hline 1. Almacenamiento & 1. Colocación de la orden & 1. Pérdida de ventas \\
\hline 2. Seguros & 2. Manejo de materiales & $\begin{array}{l}\text { 2. Disminución cuentas por } \\
\text { cobrar }\end{array}$ \\
\hline 3. Costo de capital & 3.Carga de la producción & $\begin{array}{l}\text { 3. Desorganización de } \\
\text { programas de producción }\end{array}$ \\
\hline 4. Obsolescencia & & \\
\hline
\end{tabular}

El comportamiento de estos costos los podemos observar en el siguiente gráfico.

Costos para ordenar y mantener inventarios (\$)

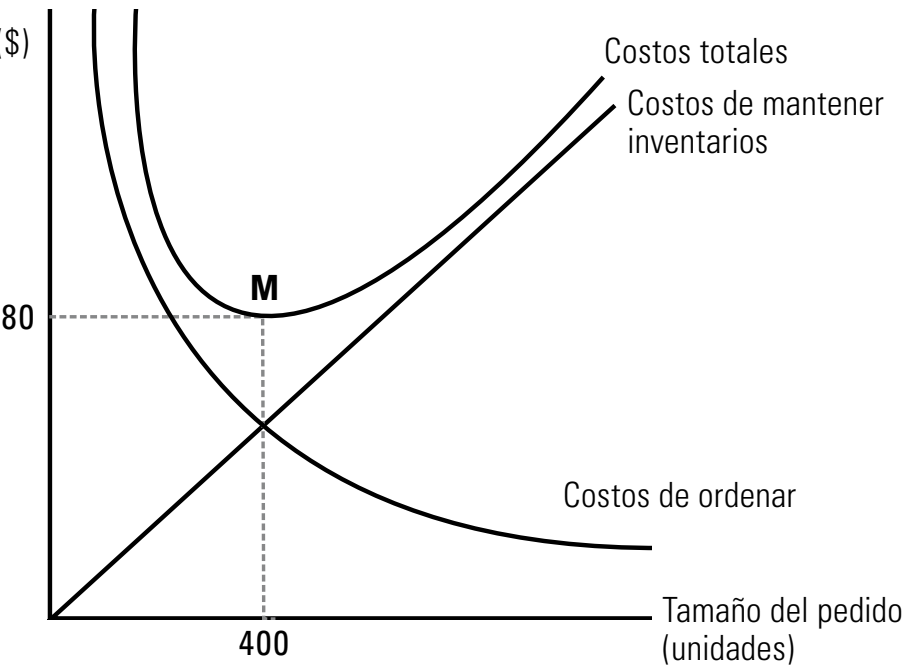


A medida que el tamaño del pedido crece, nuestros costos para mantener inventarios también aumentan ya que tenemos mayor inventario en existencia. Por supuesto, si efectuáramos pedidos más grandes, no nos veríamos obligados a pedir con mucha frecuencia y nuestros costos totales por ordenar disminuirán. La combinación más apropiada entre ambos se puede apreciar al examinar la curva del costo total. En el gráfico en el punto $\mathbf{M}$, hemos equilibrado las ventajas y desventajas de los respectivos costos. Cuando se trate de pedidos grandes, los costos para mantener los inventarios serán excesivos, en tanto que los pedidos pequeños implicarán efectuar pedidos en forma constante lo cuál nos ubicará en la parte más elevada de la curva de costos de ordenar.

Entonces surge la pregunta, ¿cómo podremos determinar el punto mínimo (M) dentro de la curva de costos totales? Para contestarla utilizaremos la fórmula del lote óptimo de compra.

$$
\mathrm{Q}=\sqrt{\frac{2 V C_{0}}{C_{m}}}
$$

Donde:

Qc = lote óptimo de compra

$\mathrm{V}=$ ventas

$\mathrm{Co}=$ costos de ordenar por pedido

$\mathrm{Cm}=$ costos de mantenimiento de inventario

Supongamos que una empresa piensa vender 2.000 unidades y que la colocación de cada pedido tiene un costo de $\$ 8$, siendo el precio de cada unidad de $\$ 1$, con un costo de mantenimiento de inventario del $20 \%$. Si reemplazamos los valores tendremos:

$$
\begin{array}{r}
Q C=\sqrt{\frac{2 \times 2000 \times \$ 8}{\$ 0,20}} \\
Q c=\sqrt{\frac{32.000}{\$ 0,20}}
\end{array}
$$

Qc $=400$ unidades

Los costos totales y los costos para mantener inventario se calcularon de la siguiente forma:

$\begin{aligned} \text { 1. Costos de ordenar } & =\frac{2000 \text { unidades }}{400 \text { unidades }}=5 \text { pedidos } \\ & =5 \text { pedidos } \text { a } 8 \text { por pedidos }=\$ 40\end{aligned}$

2. Costos para mantener los inventarios $=$ inventario promedio expresado en unidades $x$ costo para mantener inventario por unidad

$$
=200 \times \$ 0,20=\$ 40
$$

\begin{tabular}{lr} 
3. Costos de ordenar & $\$ 40$ \\
Costos para mantener & $\$ 40$ \\
\cline { 2 - 2 } Costos totales & $\$ 80$
\end{tabular}

El tamaño óptimo de pedido es de 400 unidades. Sobre el supuesto que consumamos inventario en forma constante durante el año, nuestro inventario promedio en existencia será de 200 unidades. El punto $M$ del gráfico coincide con el costo total de $\$ 80$ para un tamaño de pedido de 400 unidades. En ningún otro punto podremos esperar alcanzar los costos más bajos.

Con respecto al control del inventario, si bien generalmente no está bajo la dirección del administrador financiero, tiene asociado costos financieros. Por lo tanto, es importante controlar el inventario a un 
costo mínimo, y hacer un adecuado control del inventario para obtener una producción uniforme y ordenada para evitar costosos faltantes.

Las empresas deben almacenar y vigilar miles de distintos artículos en inventario. Esto conduce a preguntarnos ¿qué artículos deben ser más rígidamente controlados?

El sistema ABC para control de inventario es un método para asignar diferentes grados de control a los artículos del inventario. Estos se dividen en tres categorías, sobre la base de sus valores de inversión, con relación al valor total del inventario de la empresa. Generalmente los artículos A son los más costosos y constituyen el mayor porcentaje sobre la inversión total (70\% aproximadamente), pero a su vez, constituyen la menor proporción de artículos (20\% aproximadamente). Estos artículos deben ser meticulosamente controlados. Los items $\mathbf{B}$ generalmente constituyen el 30\% del inventario y solo el 20\% de la inversión, por lo que se efectuará un control promedio. En cambio, la categoría $\mathbf{C}$ comprende el 50\% de los artículos y solamente el $10 \%$ de la inversión total, por consiguiente solo se hará un control por muestreos.

De acuerdo con lo dicho, las rotaciones más rápidas del inventario reducen los requerimientos de financiamiento de capital de trabajo. Al mismo tiempo, el administrador financiero debe intentar reducir los costos asociados con el inventario de la empresa. Los modelos de inventario de seguridad pueden usarse con los de la cantidad económica de pedido. Las existencias de seguridad en el inventario proporcionan un importante seguro contra las interrupciones de la producción causadas por los faltantes y los agotamientos de productos terminados que resultan de pérdidas de ventas.

Debemos tener en cuenta que la inversión en inventarios es costosa. Dicha inversión y los riesgos asociados de pérdida son tan grandes que el administrador financiero debe concentrar la atención en su manejo. Los administradores financieros deben tener muy presente el impacto que los métodos de valuación de inventarios (LIFO, FIFO, PPP) tienen sobre los resultados y los balances generales.

\section{POLÍTICA DE FINANCIAMIENTO}

\section{(colaboración Dr. Miguel Ángel Cuello)}

Las decisiones de financiamiento tienen como finalidad contribuir a la concreción del objetivo del administrador financiero. Ante esta situación deberíamos definir dos aspectos vitales que afectan, según cuál sea su elección, a la administración de los activos de trabajo y por consiguiente, a la rentabilidad de la firma. Ellos son:

\section{Elección del enfoque de cobertura}

2. Elección del fondeo

\section{ENFOQUES DE COBERTURA}

La elección de la modalidad de cobertura guarda una relación directa con el grado de permanencia temporal de los activos en un caso o con los vencimientos de las obligaciones en otro.

\section{a) Temporalidad de la inversión}

Los estados contables de la empresa son una de las herramientas con las que el administrador financiero cuenta para obtener información que lo ayudará en el 
logro de la maximización del valor de la firma.

La clasificación contable de los activos en corrientes y no corrientes, nos brinda la magnitud de los activos de corto y largo plazo. Considerando que el activo representa la inversión total de la empresa, podemos decir que el balance nos separa la inversión de corto plazo de la inversión fija.

El enfoque de cobertura temporal de la inversión se basa en vincular los activos y su correspondiente financiación en función de su grado de permanencia, de tal manera que el activo corriente se financia con deudas de corto plazo y los activos no corrientes con fuentes de fondos de largo plazo.

Se produce un calce entre la inversión y su fondeo en relación directa al aspecto temporal de los activos.

Se deben considerar algunas circunstancias que amplían y profundizan el análisis de este enfoque, acercándolo a la realidad, ya que el desarrollo de la operatoria normal de la empresa, hace imposible ajustar exactamente la inversión de corto y largo plazo con sus correspondientes fuentes de fondos de la misma madurez.

La práctica profesional nos muestra que las inversiones no corrientes se financian con una porción de fondos de largo plazo (pasivo no corriente y fondos propios), mientras que el excedente de estos fondos financia al capital de trabajo neto; es decir que la diferencia del activo corriente y el pasivo corriente, se financia con fondos de largo plazo. De esta manera puede observarse cómo parte del capital propio soporta las variaciones que se producen en el ciclo operativo de la firma (capital de trabajo).
Otro aspecto que nos muestra la realidad es que todos los activos no guardan el mismo grado de permanencia. Teniendo en cuenta el ciclo operativo de la empresa (dinero-mercadería-dinero), podemos ver cómo ciertas partidas que se encuentran clasificadas como corrientes, en realidad adquieren las características de activos inmovilizados. Podemos citar, a modo de ejemplo, que una porción de los inventarios está formado por el stock mínimo, que adquiere la característica de permanente, aunque pertenezca al activo corriente. Es decir que el rubro desde un punto de vista financiero tiene una porción permanente y otra fluctuante; la permanente es necesaria para el desarrollo del ciclo operativo de la empresa, la fluctuante varía según la evolución de este ciclo operativo.

Similares características pueden encontrarse en otros rubros corrientes, como ser el saldo mínimo de caja (disponibilidades).

Cabe la aclaración de que en las deudas corrientes existe también la calificación de permanentes y fluctuantes.

Considerando estas circunstancias tendremos al activo total compuesto de la siguiente forma:

Activos corrientes fluctuantes

+ Activos corrientes permanentes

Activos Corrientes

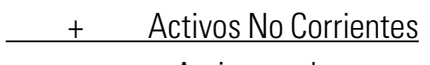

Activo total

Ante esta situación en este enfoque de cobertura temporal, la financiación de la inversión sería la siguiente: 


\begin{tabular}{|c|c|}
\hline Inversión & Financiación \\
\hline Activos corrientes fluctuantes & Fondos de corto plazo \\
\hline Activos corrientes permanentes & Fondos de largo plazo \\
\hline Activos no corrientes & Fondos de largo plazo \\
\hline
\end{tabular}

Como puede observarse los activos corrientes permanentes y los no corrientes se financian con fondos de largo plazo, considerando dentro de ellos a los fondos propios, los pasivos no corrientes y los pasivos corrientes permanentes; es decir, que las necesidades de fondos permanentes se deben financiar con fondos provenientes de financiaciones de largo plazo.

En tanto que las necesidades transitorias (activos corrientes fluctuantes) se deben financiar con fondos de corto plazo.

Además debe tenerse en cuenta que la financiación de activos fluctuantes con fondos de largo plazo provocará en ciertos momentos excesos de fondos que afectarán la rentabilidad, debido a que se generan intereses por fondos que no se utilizan. Inversamente si se financian inversiones permanentes con fondos provenientes de corto plazo, se incrementa el riesgo de no poder financiar las inversiones o tener que pagar costos superiores a los del mercado para cubrir las necesidades financieras.

El efecto generado por el riesgo es muy importante, ya que en condiciones de certidumbre es factible lograr aparear las necesidades con el financiamiento, pero a medida que el riesgo crece se comienzan a utilizar "colchones financieros", que reducen el riesgo, pero que al mismo tiempo afectan la rentabilidad.

Como observamos en este enfoque, la decisión sobre el monto y la composición de los activos corrientes y sus fuentes de financiamiento implican una compensación entre el riesgo y la rentabilidad.

En el gráfico que sigue podemos apreciar que las fluctuaciones de corto plazo que aparecen en la parte superior serían financiadas con pasivos de corto plazo.

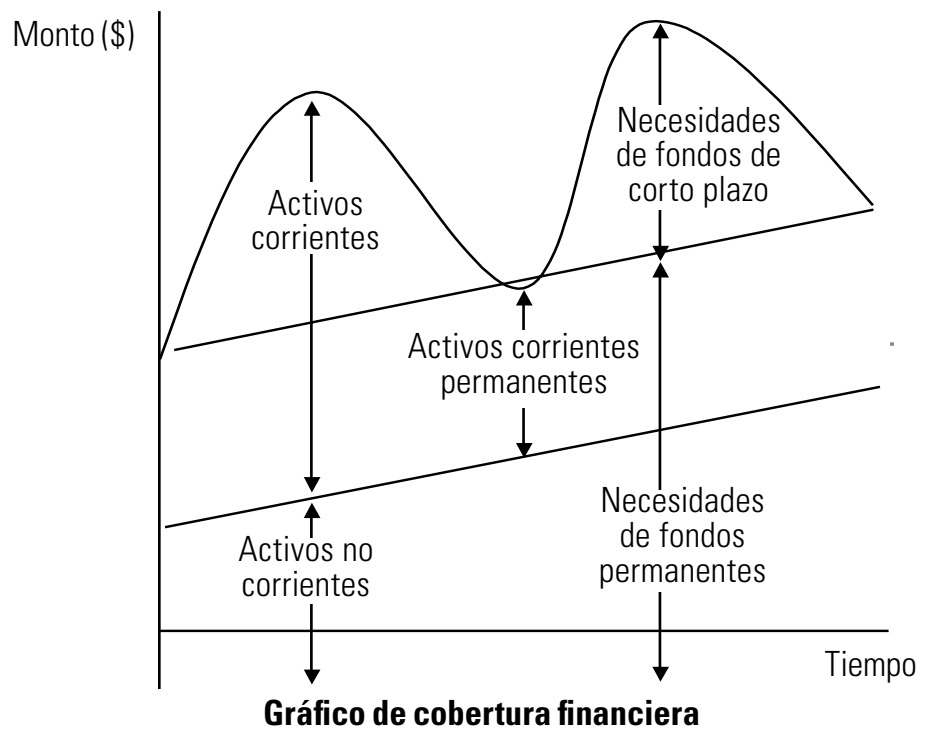


Financiar requerimientos de corto plazo con pasivos de largo plazo obligaría a pagar intereses por mantener fondos durante los períodos en que no son necesarios.

Los activos fijos y la parte permanente de los activos corrientes se financiarán con pasivos de largo plazo, con patrimonio y con el componente permanente de los pasivos corrientes. En realidad, muchas veces, la inversión en activos fijos es financiada parcialmente por pasivos de largo plazo y parcialmente con fondos propios, aunque a veces también el saldo se financia con capital de trabajo neto.

\section{b) Vencimiento de las deudas}

Esta modalidad consiste en buscar la sincronización entre los vencimientos de las obligaciones de la empresa y los ingresos futuros de fondos que se generan en ella. Como puede observarse este enfoque es netamente financiero, sin necesidad de datos contables, ya que se necesita conocer los flujos de fondos futuros de la empresa; es decir, un manejo de lo percibido y no del concepto contable del devengamiento.

La empresa debe contar con un buen sistema de información, ya que los flujos futuros de fondos, tanto cobros como pagos, tienen un carácter dinámico, debido a los cambios y movimientos que se producen durante el ciclo operativo de la empresa.

Es fundamental trabajar con un Programa de Vencimientos de las deudas; de allí la necesidad de contar con información rápida y precisa; ya que este enfoque intenta lograr que los pagos que debe efectuar la empresa se produzcan con fecha posterior a sus cobros; es decir que los flujos futuros de ingresos sean anteriores a los flujos futuros de egresos, con lo cual se dispondrían de los fondos necesarios para afrontar las obligaciones. Cabe aclarar que además de la temporalidad de los vencimientos debe tenerse en consideración los montos por cobrar y pagar, y alcanzar los fondos por cobrar para la cancelación de las futuras erogaciones.

El factor riesgo tiene incidencia importante en este enfoque, ya que en condiciones de certeza lo ideal es buscar una exacta sincronización entre los ingresos y los egresos futuros, pero en condiciones de incertidumbre el riesgo aumenta, ante lo cual pueden producirse fluctuaciones en los flujos de fondos que afectan los importes pronosticados. Una alternativa ante esta situación, es trabajar creando un margen de seguridad que permita absorber las fluctuaciones de los flujos.

El margen de seguridad consiste, al igual que en el enfoque temporal de la inversión, en constituir una reserva de fondos, con lo cual se afecta la rentabilidad. Este margen consiste en alargar o reducir el programa de vencimientos de las deudas. Cuanto más prolongado es el programa, menor es el riesgo asumido, mientras que cuanto más breve es el programa, mayor es el riesgo de la empresa por no honrar sus vencimientos.

En el caso de aumentar el margen de seguridad, se puede producir una pérdida según cuál sea el costo de los fondos, pagando intereses por períodos en que los fondos no son necesarios.

La estimación del margen de seguridad va a depender de las preferencias que estipule el administrador financiero, es decir, que la gerencia determinará el plazo entre los flujos de fondos probables y los vencimientos de las deudas. 
Esta decisión establecerá la proporción de deudas corrientes y no corrientes que mantendrá la estructura del pasivo.

\section{ElECCIÓN DEL FONDEO}

La elección de la fuente de fondos para financiar el capital de trabajo depende de la modalidad de enfoque de cobertura que se elija. No obstante, los fondos pueden provenir de endeudamiento con terceros (pasivos) o fondos propios (patrimonio neto).

\section{a) Fondos propios}

Los fondos propios se encuentran registrados contablemente en el patrimonio neto del balance de la empresa, mientras que, a partir de un concepto financiero, podemos decir que son aquellos fondos que se originan en el aporte societario, como los que se generan en la operatoria normal de la empresa, como las utilidades no distribuidas, reservas (legales, optativas) y los fondos provenientes de las cargas no erogables (depreciaciones, previsiones).

Si nos ubicamos temporalmente en el nacimiento de una empresa, podemos decir que los fondos propios forman el capital de trabajo "original" de la misma, ya que el aporte de los propietarios, excepto que fuesen bienes de uso, financian los demás activos (corrientes). Al no existir en ese momento otra fuente de financiación se puede aseverar que el aporte de los socios es la única fuente de fondos del capital de trabajo en el inicio de las actividades de la firma.

Cuando la empresa comienza a operar se van produciendo movimientos normales de su ciclo operativo que generan la necesidad de nuevos fondos, ante lo cual se debe recurrir a otras fuentes de finan- ciación, o a nuevos aportes de fondos por parte de los propietarios.

Este tipo de fondeo podemos clasificarlos temporalmente como de largo plazo, característica esencial para financiar los activos permanentes (corrientes y no corrientes) y para no tener problemas de necesidades de financiación durante el desarrollo de la actividad normal empresaria.

Es importante determinar el costo para este tipo de fondos, que en algunos componentes podrá ser costo de oportunidad y en otros costo explícito (por ejemplo, una nueva emisión de acciones).

Otro aspecto que tiene incidencia sobre los fondos propios es la decisión de distribución de dividendos, ya que esta afecta en forma directa la magnitud de los fondos; en este caso se produce una controversia entre las decisiones de financiación y las de distribución de utilidades, que debe ser salvada por el administrador financiero, para no tener problemas de disminución del capital de trabajo ni tampoco con la retribución a los accionistas por su aporte a la firma.

\section{b) Fondos de terceros}

Los fondos provenientes de la financiación provista por terceros a la empresa forman el pasivo del balance, desde un punto de vista temporal estos fondos se clasifican en corrientes y no corrientes.

El pasivo no corriente junto con el patrimonio neto forman el total de fondos de largo plazo con que cuenta la empresa, mientras que el pasivo corriente representa los fondos de corto plazo; la conjunción de los diferentes tipos de financiación nos dará como resultado la estructura de financiamiento de la firma. 
Como se citó anteriormente, la elección de la fuente de fondos va a depender de la modalidad del tipo de cobertura que se elija. Normalmente los fondos provenientes del fondeo de largo plazo, tanto propios como de terceros, financian la inversión fija y los activos corrientes permanentes, lo cual significa que financian el capital de trabajo neto (excedente del activo corriente sobre los pasivos corrientes). La importancia de esta situación radica en que los activos corrientes permanentes son los necesarios para el desarrollo del ciclo operativo de la empresa, con lo cual al fondearse con fuentes de largo plazo, se evitan trastornos en la operatoria.

Los fondos de corto plazo, al igual que el activo corriente, se dividen en permanentes y fluctuantes. Conocer la composición de cada uno de ellos es relevante, ya que los pasivos corrientes permanentes conjuntamente con el fondeo de largo plazo deben cubrir las necesidades de financiación de los activos permanentes (activos corrientes permanentes y activos inmovilizados).

Los fondos de terceros transitorios (fluctuantes) financian las necesidades transitorias que se producen durante el desarrollo del ciclo operativo de la firma.

Para determinar la mezcla de fondos que se van a utilizar en la financiación de los activos de trabajo es vital conocer en la forma más precisa (ya que la certeza es prácticamente imposible), el ciclo dineromercadería-dinero, la secuencia temporal de la operatoria normal de la empresa. Hay que conocer la cantidad de etapas que componen el ciclo, la duración de cada una de ellas y las superposiciones de procesos (solapamientos) que se producen; de la misma forma conocer las estacionalidades a las que pudiera estar expuesta la empresa, ya sea por la necesidad de insumos o materia prima, o por las características del bien que se produce o comercializa. El no conocimiento de una parte o de todo el ciclo, puede llevar a problemas de una mala selección de fuentes de financiación, ya que si tomamos fondos de largo plazo para financiar necesidades temporarias o fluctuantes, se va a producir, en algún momento, un sobrante de fondos que generará una disminución de la rentabilidad.

Al igual que con los fondos propios, es de suma importancia la determinación del costo de financiación de los fondos de terceros. Asimismo se debe tener conocimiento del comportamiento del mercado de capitales y financiero, ya que dependiendo del tipo de financiación que se necesite, se puede recurrir a estos mercados para lograrla; en el caso de fondos propios mediante la emisión de nuevas acciones (mercado de capitales), si son financiación externa a la empresa, se pueden colocar obligaciones negociables para obtener fondos a largo plazo (mercado de capitales) y las necesidades de mediano y largo plazo se pueden atender con los instrumentos de financiación que se ofrecen en el mercado financiero.

La empresa cuenta además con fondos de corto plazo que provienen de la financiación que nos brindan los proveedores de la empresa y que financian los activos corrientes. Un manejo eficiente de estos fondos puede proveer a la empresa de una porción importante de financiación de sus activos corrientes.

Recordemos que según la mezcla de financiación y los costos de cada una de las fuentes de fondos, se establecerá el costo 
Un buen control de inventarios nos va a permitir también tener sobre control el equilibrio entre la liquidez y la rentabilidad. promedio ponderado del capital, que es el estándar mínimo que aceptará la empresa para aprobar las inversiones futuras.

\section{CONCLUSIONES}

De acuerdo con lo visto en el presente trabajo y como se menciona en el título, la administración del capital de trabajo debería ser considerada por los administradores financieros como un aspecto imprescindible para llevar adelante el objetivo básico de los mismos que es "maximizar la riqueza de los accionistas”.

A su vez se deberá tener en cuenta que la misma se consigue entre otras cosas, buscando un equilibrio entre la liquidez y la rentabilidad, dado que dichas funciones son contrapuestas entre sí, o sea, a mayor tenencia de fondos líquidos la rentabilidad será menor dado que se pierden oportunidades de colocar dichos fondos en inversiones rentables o en el mismo ciclo económico de la empresa; y viceversa, si sacrifico liquidez para conseguir mayor rentabilidad, puede ocurrir que en determinados momentos, necesite cubrir desfases transitorios del ciclo económico teniendo que recurrir al endeudamiento cuyo resultado terminará afectando la rentabilidad. Parece obvio, pero merece aclararse que en el equilibrio de ambas funciones juega un papel fundamental el riesgo, tanto económico (contribución marginal) como financiero.

Por lo tanto, en contextos con restricciones tanto económicas como financieras, la administración del capital de trabajo se transforma en uno de los focos principales de la gestión financiera. Puesto que, para conservar el equilibrio anteriormente men- cionado entre liquidez y rentabilidad, el administrador financiero deberá verificar la tenencia de activos, tanto en su volumen como en la permanencia de los mismos.

También deberá profundizar la gestión de las cobranzas, buscando que el ciclo operativo pueda ser cumplido sin tener que recurrir a endeudamientos adicionales a los previstos. Esto también es válido para el pasivo, en donde se deberá buscar un apareamiento de los vencimientos con los términos normales de cobranza.

Con referencia a los inventarios, el control de los mismos deberá hacerse en forma bastante periódica, en especial en lo que respecta al control de bienes de baja o casi nula rotación y obsoletos, buscando optimizar el uso de los mismos, dado que afectan en forma directa al resultado operativo.

A su vez, un buen control de inventarios nos va a permitir también tener sobre control el equilibrio entre la liquidez y la rentabilidad.

Si se focaliza la atención en los puntos descritos buscando minimizar las inmovilizaciones de fondos, optimizando las rotaciones de cuentas de cobro e inventarios $y$ con un enfoque de cobertura financiera que permita mantener fondos durante los períodos en que no son necesarios, seguramente se conseguirá que la empresa no pase sobresaltos ante restricciones del contexto.

\section{CASO PRÁCTICO}

La evolución del activo de la empresa Isabel S. A. para el primer cuatrimestre del 2000, es la siguiente: 


\begin{tabular}{|l|c|c|c|c|}
\hline \multicolumn{1}{|c|}{ Rubros } & Enero & Febrero & Marzo & Abril \\
\hline Disponibilidades & 1.890 & 1.470 & 2.100 & 1.650 \\
\hline Créditos comerciales & 29.300 & 22.500 & 32.100 & 26.700 \\
\hline Bienes de cambio & $\underline{65.430}$ & $\underline{55.250}$ & $\underline{71.450}$ & $\underline{67.230}$ \\
\hline Activo corriente & 96.620 & 79.220 & 105.650 & 95.580 \\
\hline Bienes de uso & $\underline{10.375}$ & $\underline{10.250}$ & $\underline{10.125}$ & $\underline{10.000}$ \\
\hline Activo no corriente & $\underline{10.375}$ & $\underline{10.250}$ & $\underline{10.125}$ & $\underline{10.000}$ \\
\hline Activo total & 106.995 & 89.470 & 115.775 & 105.580 \\
\hline
\end{tabular}

Adicionalmente se informa que:

- El saldo mínimo de caja establecido por la gerencia es de $\$ 800$.

- El stock mínimo de inventarios determinado es de $\$ 35.000$.

- Se estimó un saldo inmovilizado de cuentas a cobrar de $\$ 15.000$.
- La depreciación mensual de los bienes de uso asciende a \$ 125 .

1) Sobre la base de la información disponible vamos a proceder a reclasificar los rubros del activo corriente según su grado de permanencia

\begin{tabular}{|c|c|c|c|c|}
\hline Rubros & Enero & Febrero & Marzo & Abril \\
\hline \multicolumn{5}{|l|}{ Disponibilidades } \\
\hline Transitorias & 1.090 & 670 & 1.300 & 850 \\
\hline Permanentes & 800 & 800 & 800 & 800 \\
\hline \multicolumn{5}{|l|}{ Créditos comerciales } \\
\hline Transitorios & 14.300 & 7.500 & 17.100 & 11.700 \\
\hline Permanentes & 15.000 & 15.000 & 15.000 & 15.000 \\
\hline \multicolumn{5}{|l|}{ Bienes de cambio } \\
\hline Transitorios & 30.430 & 20.250 & 36.450 & 32.230 \\
\hline Permanentes & 35.000 & 35.000 & 35.000 & 35.000 \\
\hline
\end{tabular}

La reclasificación consistió en separar, para cada uno de los rubros del activo corriente, la porción correspondiente a las partidas transitorias de aquellas que pertenecen a las permanentes.
Podemos observar en el cuadro siguiente cómo queda reestructurado el activo corriente:

\begin{tabular}{|c|c|c|c|c|}
\hline \multicolumn{1}{|c|}{ Rubros } & Enero & Febrero & Marzo & Abril \\
\hline Act. corr. transitorios & 45.820 & 28.420 & 54.820 & 44.780 \\
\hline Act. corr. permanentes & $\underline{50.800}$ & $\underline{50.800}$ & $\underline{50.800}$ & $\underline{50.800}$ \\
\hline Activo corriente & 96.620 & 79.220 & 105.650 & 50.800 \\
\hline
\end{tabular}


2) La composición del activo considerando su grado de permanencia temporal sería la siguiente:

\begin{tabular}{|c|c|c|c|c|}
\hline Rubros & Enero & Febrero & Marzo & Abril \\
\hline Act. corr. transitorios & 45.820 & 28.420 & 54.850 & 44.780 \\
\hline Act. corr. permanentes & 50.800 & 50.800 & 50.800 & 50.800 \\
\hline Activos no corrientes & $\underline{10.375}$ & $\underline{10.250}$ & $\underline{10.125}$ & $\underline{10.000}$ \\
\hline ACTIVO & 106.995 & 89.470 & 115.775 & 105.580 \\
\hline
\end{tabular}

3) Mediante el gráfico podemos observar la evolución de los rubros en el período analizado:

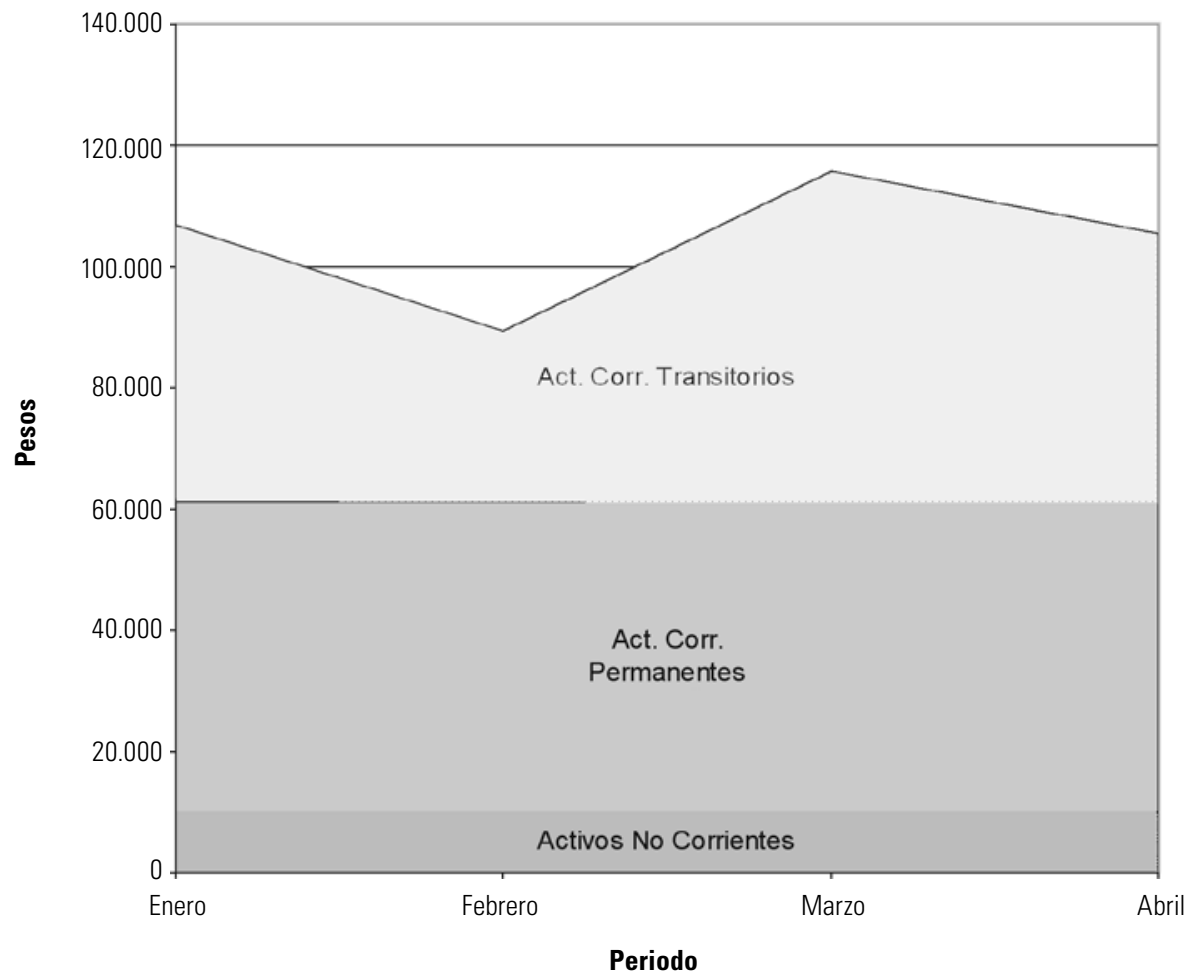

En el gráfico puede observarse que los activos corrientes permanentes como los activos no corrientes, prácticamente no sufren variaciones durante el período (cuatrimestre), ya que el único rubro que se modifica es el de los bienes de uso, por efecto de las depreciaciones; mientras que el activo corriente transitorio sufrió variaciones que se produjeron como consecuencia del desarrollo de la operatoria de la empresa. 
Las necesidades de fondos generadas por los rubros permanentes (tanto los activos corrientes como los no corrientes), deben atenderse con financiación de largo plazo (fondos propios, pasivos no corrientes o pasivos corrientes permanentes), mientras que aquellas necesidades de fondos transitorias (activos corrientes transitorios) deben atenderse con pasivos corrientes transitorios.

\section{BIBLIOGRAFÍA}

- Basagaña y colab. Administración Financiera I y II. Buenos Aires: Ed. Macchi, 1984.

- Brealey, R y Myers S. Fundamentos de Financiación Empresarial. Madrid: Mc Graw Hill, 1998.

- Brealey, R y Myers S . Principios de Finanzas Corporativas. Madrid: Mc Graw Hill, 2003.

- Brealey, R y Myers S. Principios de Dirección Financiera. Madrid: Mc Graw Hill, 1996.

- Drimer R. Finanzas de Empresas. Buenos Aires: Editorial Osman Buyatti, 2001.

- Messuti D. Temas de Administración. Buenos Aires: Ed.Macchi.

- Mondino D y Pendas E. Finanzas para Empresas Competitivas. Buenos Aires: Granica, 2005.

- Pascale R. Decisiones Financieras. Buenos Aires: Ed. Macchi, 1992.

- Ross, S.A.; Westerfield, R.W. y Jaffe J.F. Finanzas Corporativas. Madrid: Irwin/Mc Graw Hill, 1995.

- Sapetnitzky y colab. Administración Financiera de las Organizaciones. Buenos Aires: Ed. Macchi, 2003.

- Suarez, A. Decisiones Óptimas de Inversión y Financiamiento. Madrid: Ed. Pirámide, 1980.

- Van Horne. Administración Financiera. México: Ed. Prentice Hall, 1999.

- Van Horne. Administración Financiera. Ediciones de Contabilidad Moderna. Prentice Hall, 1971.

- Van Horne y Wachowicz. Fundamentos de Administración Financiera. México: Ed. Prentice Hall, 1999.

- Weston F y Copeland T. Finanzas en Administración. Madrid: Mc Graw Hill, 1983.

- Macario Alberto. "Magnitud y estructura de inversiones en las empresas", en São Paulo: RAE (Revista de Administração de Empresas), núm. 12, pág. 361.

- Macario Alberto. "El capital de trabajo: sus efectos económicos y financieros”, en São Paulo: RAE (Revista de Administração de Empresas), núm.13, pág.11.

- Del Águila Jorge y otros. “Optimización del riesgo en la asignación crediticia”, en São Paulo: RAE (Revista de Administração de Empresas), núm. 7, pág. 643.

- Martino Roque. "Administración de cuentas a cobrar”, en São Paulo: RAE (Revista de Administração de Empresas), núm. 11, pág. 249.

- Pecheny David. "Comportamiento de un modelo de control de stock en condiciones de inflación”, en São Paulo: RAE (Revista de Administração de Empresas), núm 1. pág.943.

- Slaybaugh Charles. "Administración de inventarios”, en São Paulo: RAE (Revista de Administração de Empresas), núm. 6, pág. 795. 